\title{
A VALUE FOR GAMES RESTRICTED BY AUGMENTING SYSTEMS*
}

\author{
E. $\operatorname{ALGABA}^{\dagger}$, J. M. BILBAO ${ }^{\dagger}$, AND M. SLIKKER
}

\begin{abstract}
This paper deals with cooperative games in which only certain coalitions are allowed to form. There have been previous models developed to confront the problem of nonfeasible coalitions. Games restricted by a communication graph are games in which the feasible coalitions are those that induce connected subgraphs. Another type of model is determined by the positions of the players in a so-called permission structure. In this paper, the restrictions to the cooperation are given by a combinatorial structure called an augmenting system which generalizes antimatroid structure and the system of connected subgraphs of a graph. Furthermore, the class of augmenting systems includes the conjunctive and disjunctive systems derived from a permission structure. The value $\alpha$ is a generalization of the Myerson value for games restricted by graphs and the Shapley value for games restricted by permission structures. The main results of the paper are the characterization of the value $\alpha$ for augmenting structures by using component efficiency, loop-null, and balanced contributions, and another characterization by consistency of this value. Furthermore, we implement a direct algorithm to compute this value by using the outputs of the original game.
\end{abstract}

Key words. augmenting system, consistency, Shapley value

AMS subject classification. $91 \mathrm{~A} 12$

DOI. $10.1137 / 080719170$

1. Introduction. Cooperative games under combinatorial restrictions are a type of cooperative game in which the players have restricted communication possibilities which are defined by a combinatorial structure. The first model in which the restrictions are defined by the connected subgraphs of a graph was introduced by Myerson [10]. Since then, many other situations where players have communication restrictions have been studied in cooperative game theory. Contributions on graphrestricted games include Owen [12], Borm, Owen, and Tijs [3], and Hamiache [7]. In these models the possibilities of coalition formation are determined by the positions of the players in a communication graph. Another type of combinatorial structure introduced by Gilles, Owen, and van den Brink [6] and van den Brink [15] is equivalent to a subclass of antimatroids. This line of research focuses on the possibilities of coalition formation determined by the positions of the players in the so-called permission structure.

Consider a setting with four players that are almost completely connected, the only pair of players not connected directly being players 1 and 4 . A license is required to sell products, which is the way profit can be obtained in this example. Licenses are assumed to be transferable in a coalition. Initially, each one of players 1 and 4 has a license. A coalition is now called feasible if it is internally connected and has at least one license. Note that the union of two feasible coalitions with a nonempty intersection is feasible again. Moreover, for any nonempty feasible coalition $T$ and any

${ }^{*}$ Received by the editors March 25, 2008; accepted for publication (in revised form) June 4, 2010; published electronically August 17, 2010. This research was partially supported by the Spanish Ministry of Education and Science and the European Regional Development Fund under grant SEJ2006-00706 and by the FQM 237 grant of the Andalusian Government.

http://www.siam.org/journals/sidma/24-3/71917.html

${ }^{\dagger}$ Department of Applied Mathematics II, University of Seville, 41092 Sevilla, Spain (ealgaba@us. es, mbilbao@us.es).

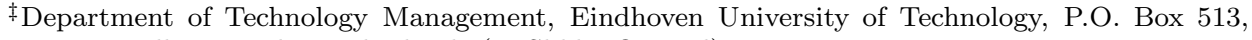
5600 MB, Eindhoven, The Netherlands (M.Slikker@tue.nl). 
of its feasible subcoalitions $S$, one can find a player that is in $T$ but not in the coalition $S$ such that the player can join to coalition $S$ and the result will be a feasible coalition again. Finally, the empty set is assumed to be feasible. In fact, these three conditions make up a combinatorial structure called an augmenting system, introduced by Bilbao [2]. The example sketched results in an augmenting system that cannot be obtained from a communication graph and which is not an antimatroid either; consequently, it does not correspond to any of the two set systems derived from permission structures. An argument similar to the one given in the former example can be derived in a four-retailer system with two warehouses. Consider four retailers which are almost completely connected except for retailers 1 and 4, who are not connected directly. A warehouse is necessary to replenish the inventory. Retailers 1 and 4 are the only ones with a warehouse. A coalition is now called feasible if it is internally connected and has at least one warehouse. Therefore, as before, this example gives way to an augmenting system which cannot be modeled by a communication graph or an antimatroid. ${ }^{1}$ We stress here that the set of augmenting systems contains, on one hand, the set of antimatroid systems, in particular the sets of conjunctive and disjunctive systems derived from a permission structure, and, on the other hand, the set of systems (connected coalitions) that can be derived from a graph. So, two important lines of research in the literature are unified.

The focus of this paper is on an allocation rule for games under augmenting systems. This rule coincides with the Myerson value for communication situations (games with a graph) and with the Shapley value for games on antimatroids. We concentrate on two characterizations. The first one uses a balanced contribution type of property, in line with the results of Myerson [11] and Slikker [14]. Our extended setting calls for the use of tools from discrete mathematics, such as the Möbius inversion formula. The second characterization uses a consistency property inspired by the results of Hart and Mas-Colell [8] for the unconstrained setting. This consistency property requires the same payoffs for players in the original setting and in a natural reduced setting (with respect to the allocation rule) with fewer players. A natural formulation of this reduced framework is in terms of restriction systems and the trace on subsets. Technical complexities following these definitions to obtain a characterization are tackled.

The setup of this paper is as follows. In section 2, we recall preliminaries on augmenting systems and related combinatorial structures, followed in section 3 by the analysis of new structural properties of augmenting systems. Sections 4 and 5 concentrate on characterizations of a value for games under augmenting systems using a balanced contributions property and a consistency property, respectively. Section 6 presents an algorithm to compute this value by using the outputs of the nonrestricted game. These results generalize, unify, and simplify the results of Myerson [10], Owen [12], Gilles, Owen, and van den Brink [6], and van den Brink [15].

2. Augmenting systems. This section is based on Bilbao [2]. It basically recalls preliminaries on augmenting systems and some concepts and results that will be used in the following. Antimatroids were introduced by Dilworth [4] as particular examples of semimodular lattices. Since then, several authors have obtained the same concept by abstracting various combinatorial situations (see Korte, Lovász, and Schrader [9]). Let $N$ be a finite set. A set system over $N$ is a pair $(N, \mathcal{F})$ where $\mathcal{F} \subseteq 2^{N}$ is a family of subsets. The sets belonging to $\mathcal{F}$ are called feasible. We will write $S \cup i$ and $S \backslash i$ instead of $S \cup\{i\}$ and $S \backslash\{i\}$, respectively.

\footnotetext{
${ }^{1}$ These examples lead to a coalition structure whose diagram is showed in Figure 1.
} 
Definition 2.1. A set system $(N, \mathcal{A})$ is an antimatroid if

(A1) $\emptyset \in \mathcal{A}$;

(A2) for $S, T \in \mathcal{A}$ we have $S \cup T \in \mathcal{A}$;

(A3) for $S \in \mathcal{A}$ with $S \neq \emptyset$, there exists $i \in S$ such that $S \backslash i \in \mathcal{A}$.

Let $(N, \mathcal{A})$ be an antimatroid and let $S, T \in \mathcal{A}$ such that $|S|<|T|$. Property A3 implies an ordering $T=\left\{i_{1}, \ldots, i_{t}\right\}$ with $\left\{i_{1}, \ldots, i_{j}\right\} \in \mathcal{A}$ for $j=1, \ldots, t$. Let $k \in\{1, \ldots, t\}$ be the minimum index with $i_{k} \notin S$. Then $S \cup i_{k}=S \cup\left\{i_{1}, \ldots, i_{k}\right\} \in$ $\mathcal{A}$ by property A2. Therefore, the definition of antimatroid implies the following augmentation property: If $S, T \in \mathcal{A}$ with $|S|<|T|$, then there exists $i \in T \backslash S$ such that $S \cup i \in \mathcal{A}$.

Convex geometries are a combinatorial abstraction of convex sets introduced by Edelman and Jamison [5].

Definition 2.2. A set system $(N, \mathcal{G})$ is a convex geometry if it satisfies the following properties:

(G1) $\emptyset \in \mathcal{G}$;

(G2) for $S, T \in \mathcal{G}$ we have $S \cap T \in \mathcal{G}$;

(G3) for $S \in \mathcal{G}$ with $S \neq N$, there exists $i \in N \backslash S$ such that $S \cup i \in \mathcal{G}$.

Next, we will recall the formal concept of an augmenting system.

DEFINITION 2.3. An augmenting system is a set system $(N, \mathcal{F})$ with the following properties:

$(\mathrm{P} 1) \emptyset \in \mathcal{F}$;

(P2) for $S, T \in \mathcal{F}$ with $S \cap T \neq \emptyset$, we have $S \cup T \in \mathcal{F}$;

(P3) for $S, T \in \mathcal{F}$ with $S \subset T$, there exists $i \in T \backslash S$ such that $S \cup i \in \mathcal{F}$.

Now, it is given the relationship between the combinatorial structures mentioned above.

Proposition 2.4. (i) An augmenting system $(N, \mathcal{F})$ is an antimatroid if and only if $\mathcal{F}$ is closed under union.

(ii) An augmenting system $(N, \mathcal{F})$ is a convex geometry if and only if $\mathcal{F}$ is closed under intersection and $N \in \mathcal{F}$.

Example. The following collections of subsets of $N=\{1, \ldots, n\}$, given by $\mathcal{F}=2^{N}$, $\mathcal{F}=\{\emptyset,\{i\}\}$, where $i \in N$, and $\mathcal{F}=\{\emptyset,\{1\}, \ldots,\{n\}\}$, are augmenting systems over $N$.

Example. In a communication graph $G=(N, E)$, the set system $(N, \mathcal{F})$ given by $\mathcal{F}=\{S \subseteq N:(S, E(S))$ is a connected subgraph of $G\}$ is an augmenting system.

Example. Gilles, Owen, and van den Brink [6] showed that the feasible coalition system $(N, \mathcal{F})$ derived from the conjunctive or disjunctive approach contains the empty set and the ground set $N$ and that it is closed under union. Algaba et al. [1] showed that the coalition systems derived from the conjunctive and disjunctive approach were identified to poset antimatroids and antimatroids with the path property, respectively. Thus, these coalition systems are augmenting systems.

Remark. Notice that augmenting systems include antimatroids and the systems of connected subgraphs of a given graph. However, the system of connected subgraphs of a communication graph is not closed under union. So, in order to unify these structures in the augmenting systems it is vital to require property (P2) to hold, which establishes that common players in two feasible coalitions will perform a very important role to turn the union of two feasible coalitions into a bigger feasible coalition.

Definition 2.5. Let $(N, \mathcal{F})$ be an augmenting system. For a feasible coalition $S \in \mathcal{F}$, we define the set $S^{*}=\{i \in N \backslash S: S \cup i \in \mathcal{F}\}$ of augmentations of $S$ and the set $S^{+}=S \cup S^{*}=\{i \in N: S \cup i \in \mathcal{F}\}$. 
Proposition 2.6. Let $(N, \mathcal{F})$ be an augmenting system. Then the interval

$$
\left[S, S^{+}\right]_{\mathcal{F}}=\left\{C \in \mathcal{F}: S \subseteq C \subseteq S^{+}\right\}
$$

is equal to $\left\{C \in 2^{N}: S \subseteq C \subseteq S^{+}\right\}$for every nonempty $S \in \mathcal{F}$.

Let $(N, \mathcal{F})$ be a set system and let $S \subseteq N$ be a subset. The maximal nonempty feasible subsets of $S$ are called components of $S$. Observe that if $(N, \mathcal{A})$ is an antimatroid, then any subset $S \subseteq N$ has a unique component given by the following operator $\operatorname{int}(S)=\bigcup\{C \in \mathcal{A}: C \subseteq S\}$. We denote by $C_{\mathcal{F}}(S)$ the set of the components of a subset $S \subseteq N$. Observe that the set $C_{\mathcal{F}}(S)$ may be the empty set. This set will play a role in the concept of a game restricted by an augmenting system.

Proposition 2.7. A set system $(N, \mathcal{F})$ satisfies property $\mathrm{P} 2$ if and only if, for any $S \subseteq N$ with $C_{\mathcal{F}}(S) \neq \emptyset$, the components of $S$ form a partition of a subset of $S$.

3. The supports of an augmenting system. Let $(N, \mathcal{F})$ be an augmenting system and let $\mathcal{G} \subseteq \mathcal{F}$. We define inductively the families

$$
\mathcal{G}^{(0)}=\mathcal{G}, \quad \mathcal{G}^{(n)}=\left\{S \cup T: S, T \in \mathcal{G}^{(n-1)}, S \cap T \neq \emptyset\right\} \quad(n=1,2, \ldots) .
$$

Notice that $\mathcal{G}^{(0)} \subseteq \mathcal{G}^{(n-1)} \subseteq \mathcal{G}^{(n)} \subseteq \mathcal{F}$, since $\mathcal{G} \subseteq \mathcal{F}$ and $\mathcal{F}$ satisfies property P2. The inductive process is finite because $\mathcal{F}$ is finite.

Definition 3.1. Let $(N, \mathcal{F})$ be an augmenting system and let $\mathcal{G} \subseteq \mathcal{F}$. We define the closure $\overline{\mathcal{G}}$ by $\overline{\mathcal{G}}=\mathcal{G}^{(k)}$, where $k$ is the smallest integer such that $\mathcal{G}^{\left({ }^{k+1)}\right.}=\mathcal{G}^{(k)}$.

Example. Let us consider $N=\{1,2,3,4\}$ and the family given by

$$
\begin{aligned}
\mathcal{F}= & \{\emptyset,\{1\},\{4\},\{1,2\},\{1,3\},\{2,4\},\{3,4\}, \\
& \{1,2,3\},\{1,2,4\},\{1,3,4\},\{2,3,4\}, N\} .
\end{aligned}
$$

For the family $\mathcal{G}=\{\emptyset,\{1\},\{4\},\{1,2\},\{1,3\},\{2,4\},\{3,4\}\}$, note that

$$
\begin{aligned}
& \mathcal{G}^{(1)}=\mathcal{G} \cup\{\{1,2,3\},\{1,2,4\},\{1,3,4\},\{2,3,4\}\}, \\
& \mathcal{G}^{(2)}=\mathcal{G}^{(1)} \cup\{N\} \text { and } \mathcal{G}^{(2)}=\overline{\mathcal{G}}=\mathcal{F} .
\end{aligned}
$$

Let $(N, \mathcal{F})$ be an augmenting system. Then there can be feasible coalitions which can be written as the union of two feasible coalitions with a nonempty intersection. So, we can consider the following set:

$$
R(\mathcal{F})=\{R \in \mathcal{F}: R=S \cup T, S \neq R, T \neq R, S, T \in \mathcal{F}, S \cap T \neq \emptyset\} .
$$

Note that $R(\mathcal{F})$ is composed of those feasible coalitions which can be written as the union of two distinct feasible coalitions with a nonempty intersection.

Definition 3.2. Let $(N, \mathcal{F})$ be an augmenting system. The elements of the set $B(\mathcal{F})=\mathcal{F} \backslash R(\mathcal{F})$ are called supports of $(N, \mathcal{F})$.

By construction, the set $B(\mathcal{F})$ is unique, nonempty if $\mathcal{F}$ is nonempty, and it satisfies the following properties.

1. If $\emptyset \in \mathcal{F}$, then $\emptyset \in B(\mathcal{F})$.

2. If $\{i\} \in \mathcal{F}$ for some $i \in N$, then $\{i\} \in B(\mathcal{F})$.

3. If $S \in \mathcal{F}$ is a minimal element in $(\mathcal{F}, \subseteq)$, then $S \in B(\mathcal{F})$.

4. If $S \in \mathcal{F}$ and $|S| \leq 2$, then $S \in B(\mathcal{F})$. 


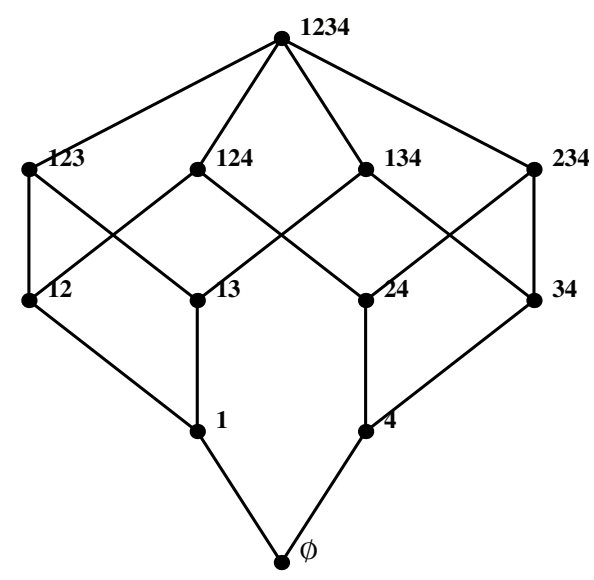

FIG. 1. The augmenting system $(N, \mathcal{F})$.

Next, we obtain the following characterization of the set of supports.

Proposition 3.3. Let $(N, \mathcal{F})$ be an augmenting system and $B(\mathcal{F})$ the set of its supports. Then $B(\mathcal{F})$ is the minimal subset of $\mathcal{F}$ such that $\overline{B(\mathcal{F})}=\mathcal{F}$.

Proof. We first prove that $\overline{B(\mathcal{F})}=\mathcal{F}$. We have that $\overline{B(\mathcal{F})} \subseteq \mathcal{F}$, since $B(\mathcal{F}) \subseteq \mathcal{F}$ and $\mathcal{F}$ satisfies property $\mathrm{P} 2$. In order to prove the reverse inclusion, we use induction on the number of elements of feasible coalitions in $\mathcal{F}$. Clearly, the minimal elements in $(\mathcal{F}, \subseteq)$ belong to the system of supports and hence to $\overline{B(\mathcal{F})}$. Now, suppose $F \in \overline{B(\mathcal{F})}$ for all $F \in \mathcal{F}$ with $|F|<p$. Then, given $F \in \mathcal{F}$ with $|F|=p$, we have either $F \in B(\mathcal{F})$ or $F \notin B(\mathcal{F})$. In the first case $F \in \overline{B(\mathcal{F})}$. Otherwise, $F \in D(\mathcal{F})$, and hence there are two feasible coalitions $S, T \in \mathcal{F}, S \neq F, T \neq F, S \cap T \neq \emptyset$ such that $S \cup T=\underline{F}$. By using the induction hypothesis, since $|S|<p$ and $|T|<p$, we have that $S, T \in \overline{B(\mathcal{F})}$, and property P2 implies that $F=S \cup T \in \overline{B(\mathcal{F})}$. Finally, we note that $B(\mathcal{F})$ is a minimal subset of $\mathcal{F}$ such that $\overline{B(\mathcal{F})}=\mathcal{F}$ by construction.

Example. The set system given by $N=\{1,2,3,4\}$ and

$$
\begin{aligned}
\mathcal{F}= & \{\emptyset,\{1\},\{4\},\{1,2\},\{1,3\},\{2,4\},\{3,4\}, \\
& \{1,2,3\},\{1,2,4\},\{1,3,4\},\{2,3,4\}, N\}
\end{aligned}
$$

is the augmenting system given in Figure 1 . Since $\{1,4\} \notin \mathcal{F}$ the system $(N, \mathcal{F})$ is not an antimatroid. Moreover, $\{1,2\} \cap\{2,4\}=\{2\} \notin \mathcal{F}$, and hence $(N, \mathcal{F})$ is not a convex geometry.

Notice that the feasible coalition structure shown in Figure 1 corresponds to the two examples mentioned in the introduction. The family of supports of $(N, \mathcal{F})$ is

$$
B(\mathcal{F})=\{\emptyset,\{1\},\{4\},\{1,2\},\{1,3\},\{2,4\},\{3,4\}\} .
$$

Let us consider an augmenting system $(N, \mathcal{F})$. An element $i$ of a feasible set $S \in \mathcal{F}$ is an extreme point of $S$ if $S \backslash i \in \mathcal{F}$. The set of extreme points of $S$ is denoted by $\operatorname{ex}(S)$. Note that property P3 implies A3 and hence $|\operatorname{ex}(S)| \geq 1$ for any nonempty $S \in \mathcal{F}$.

Lemma 3.4. Let $(N, \mathcal{F})$ be an augmenting system such that $\{i\} \in \mathcal{F}$ for all $i \in N$. Then

(1) every $S \in \mathcal{F}$ with $|S| \geq 2$ satisfies $|\operatorname{ex}(S)| \geq 2$;

(2) every support $B \in B(\mathcal{F})$ satisfies $|B| \leq 2$. 
Proof. (1) Suppose on the contrary that there exists $S \in \mathcal{F}$ with $|S| \geq 2$ such that $|\operatorname{ex}(S)|=1$. Then for some $i \in S$ we have $S \backslash i \in \mathcal{F}$ and $S \backslash j \notin \mathcal{F}$ for all $j \in S$ such that $j \neq i$. By hypothesis the set $\{i\} \in \mathcal{F}$, and using property P3 we obtain the chain

$$
\{i\}=S_{1} \subset S_{2} \subset \cdots \subset S_{s-1} \subset S_{s}=S,
$$

where $S_{k} \in \mathcal{F}$ and $\left|S_{k}\right|=k$ for $1 \leq k \leq s$. Since $i \in S_{s-1}$ we have $S_{s-1}=S \backslash j$ for some $j \in S, j \neq i$, in contradiction with the assumption.

(2) Suppose that $B \in B(\mathcal{F})$ is a support of $\mathcal{F}$ such that $|B|>2$. Then by (1) there exist $i, j \in B$, with $i \neq j, B \backslash i \in \mathcal{F}$, and $B \backslash j \in \mathcal{F}$. Thus

$$
(B \backslash i) \cup(B \backslash j)=B \text { and }(B \backslash i) \cap(B \backslash j)=B \backslash\{i, j\} \neq \emptyset,
$$

which contradicts the fact that $B$ is a support of $\mathcal{F}$.

THEOREM 3.5. An augmenting system $(N, \mathcal{F})$ is the system of connected subgraphs of the graph $G=(N, E)$, where $E=\{S \in \mathcal{F}:|S|=2\}$ if and only if $\{i\} \in \mathcal{F}$ for all $i \in N$.

Proof. For every $i \in N$, the subgraph of $G$ induced by $\{i\}$ is connected and hence $\{i\} \in \mathcal{F}$. Conversely, let $(N, \mathcal{F})$ be an augmenting system such that every singleton $\{i\} \in \mathcal{F}$. Let us consider the system $(N, \mathcal{G})$ of connected subgraphs of the graph $G$. If $S \in \mathcal{G}$, then the subgraph $(S, E(S))$ is connected, and hence either $S=\{i\}$ or $S$ is a union of edges with nonempty intersection. Since $E \subseteq \mathcal{F}$, property $\mathrm{P} 2$ gives $S \in \mathcal{F}$. To obtain the reverse inclusion, let $S \in \mathcal{F}$. If $|S| \leq 2$, then $S \in \mathcal{G}$. Suppose now that $|S|>2$. By Lemma 3.4(2) the nonempty supports of $\mathcal{F}$ are edges or vertices of the graph $G$. Then $S$ is a union of edges of $G$ with nonempty intersection and thus $S \in \mathcal{G}$.

4. A value for augmenting structures. In this section we will characterize an allocation rule for augmenting structures. First, we recall restricted games under augmenting systems introduced in Bilbao [2].

Definition 4.1. Let $v: 2^{N} \rightarrow \mathbb{R}$ be a cooperative game and let $(N, \mathcal{F})$ be an augmenting system. The restricted game $v^{\mathcal{F}}: 2^{N} \rightarrow \mathbb{R}$ is defined by

$$
v^{\mathcal{F}}(S)=\sum_{T \in C_{\mathcal{F}}(S)} v(T) \text { for all } S \subseteq N .
$$

Notice that for any $S \subseteq N$ such that $C_{\mathcal{F}}(S)=\emptyset$, we have $v^{\mathcal{F}}(S)=0$. If $(N, \mathcal{F})$ is the augmenting system given by the connected subgraphs of a graph $G=(N, E)$, then the game $\left(N, v^{\mathcal{F}}\right)$ is a graph-restricted game which is studied by Myerson [10] and Owen [12]. Let $(N, \mathcal{A})$ be an antimatroid. Since $\mathcal{A}$ is closed under union, the unique component of every $S \subseteq N$ satisfies

$$
C_{\mathcal{A}}(S)=\left\{\bigcup_{\{T \in \mathcal{A}: T \subseteq S\}} T\right\} .
$$

If we denote the unique element of $C_{\mathcal{A}}(S)$ by $c_{\mathcal{A}}(S)$, then the restricted game $v^{\mathcal{A}}$ : $2^{N} \rightarrow \mathbb{R}$ is defined by $v^{\mathcal{A}}(S)=v\left(c_{\mathcal{A}}(S)\right)$.

Let $\mathbb{P}$ be the set of all positive integers and let $N=\{1, \ldots, n\} \subset \mathbb{P}$. An augmenting structure is a triple $(N, v, \mathcal{F})$, where $(N, v)$ is a cooperative game $v: 2^{N} \rightarrow \mathbb{R}$ with $v(\emptyset)=0$, and $(N, \mathcal{F})$ is an augmenting system. The set of all augmenting structures 
with player set $N$ is denoted by $A S^{N}$, and the set of all augmenting structures is given by

$$
A S=\bigcup_{N \subset \mathbb{P}} A S^{N}
$$

The Shapley value of a game $(N, v)$ is the vector $\Phi(N, v) \in \mathbb{R}^{n}$ given by

$$
\Phi_{i}(N, v)=\sum_{\{S \subseteq N: i \in S\}} \frac{(s-1) !(n-s) !}{n !}(v(S)-v(S \backslash i)),
$$

where $i \in N, n=|N|$, and $s=|S|$. We consider the following allocation rule for games restricted by augmenting systems.

DEFINITION 4.2. The value $\alpha$ for the set of augmenting structures $A S$ is the function $\alpha: A S \rightarrow \bigcup_{n \geq 1} \mathbb{R}^{n}$, defined by $\alpha(N, v, \mathcal{F})=\Phi\left(N, v^{\mathcal{F}}\right)$, where $\Phi\left(N, v^{\mathcal{F}}\right) \in$ $\mathbb{R}^{n}$ is the Shapley value of the restricted game.

If $(N, \mathcal{F})$ is an augmenting system such that $\{i\} \in \mathcal{F}$ for all $i \in N$, then the value $\alpha(N, v, \mathcal{F})$ coincides with the Myerson value for games restricted by graphs.

We now describe the outcome of a cooperative game $(N, v)$ restricted by an augmenting system $(N, \mathcal{F})$. First, we consider component efficiency, a property that would be desirable for an allocation rule on the set of augmenting systems $A S$, and then we focus on the study of the value $\alpha$ for $A S$.

Definition 4.3. An allocation rule $\gamma: A S \rightarrow \bigcup_{n \geq 1} \mathbb{R}^{n}$ satisfies component efficiency if

$$
\sum_{i \in M} \gamma_{i}(N, v, \mathcal{F})=v(M)
$$

for all $(N, v, \mathcal{F}) \in A S$ and $M \in C_{\mathcal{F}}(N)$.

The components of $N$ form a partition of a subset of $N$, and we will show that the allocation rule $\alpha$ on $A S$ satisfies component efficiency.

Proposition 4.4. The value $\alpha: A S \rightarrow \bigcup_{n \geq 1} \mathbb{R}^{n}$ satisfies component efficiency on $A S$.

Proof. Let $(N, v, \mathcal{F}) \in A S$. If $N \in \mathcal{F}$, then $C_{\mathcal{F}}(N)=\{N\}$, and hence

$$
\sum_{i \in N} \alpha_{i}(N, v, \mathcal{F})=\sum_{i \in N} \Phi_{i}\left(N, v^{\mathcal{F}}\right)=v^{\mathcal{F}}(N)=v(N) .
$$

Suppose that $N \notin \mathcal{F}$. To each component $C \in C_{\mathcal{F}}(N)$ we consider the game $w^{C}: 2^{N} \rightarrow \mathbb{R}$, which is defined by

$$
w^{C}(T)=v^{\mathcal{F}}(T \cap C)=\sum_{S \in C_{\mathcal{F}}(T \cap C)} v(S) .
$$

Since

$$
C_{\mathcal{F}}(T)=\bigcup\left\{C_{\mathcal{F}}(T \cap C): C \in C_{\mathcal{F}}(N)\right\}
$$

for any coalition $T \subseteq N$, we have that

$$
v^{\mathcal{F}}=\sum_{C \in C_{\mathcal{F}}(N)} w^{C}
$$

Copyright $@$ ( ) by SIAM. Unauthorized reproduction of this article is prohibited. 
Taking this into account and using the linearity of the Shapley value, for every component $M \in C_{\mathcal{F}}(N)$ we obtain

$$
\sum_{i \in M} \Phi_{i}\left(N, v^{\mathcal{F}}\right)=\sum_{i \in M} \Phi_{i}\left(N, w^{M}\right)+\sum_{\left\{C \in C_{\mathcal{F}}(N): C \neq M\right\}}\left(\sum_{i \in M} \Phi_{i}\left(N, w^{C}\right)\right) .
$$

Since

$$
\sum_{i \in M} \Phi_{i}\left(N, w^{M}\right)=v^{\mathcal{F}}(M)
$$

and $\Phi_{i}\left(N, w^{C}\right)=0$ for all $C \neq M$ and $i \in M$, the above expression implies that

$$
\sum_{i \in M} \alpha_{i}(N, v, \mathcal{F})=\sum_{i \in M} \Phi_{i}\left(N, v^{\mathcal{F}}\right)=v^{\mathcal{F}}(M)=v(M)
$$

Let $(N, \mathcal{F})$ be an augmenting system. A player $i \in N$ is called a loop player in $(N, \mathcal{F})$ if

$$
i \notin \bigcup\left\{S \in 2^{N}: S \in \mathcal{F}\right\}=\bigcup\left\{M \in \mathcal{F}: M \in C_{\mathcal{F}}(N)\right\} .
$$

Remark. In an augmenting system derived from the system of connected subgraphs of a communication graph, every player is feasible and therefore there are no loop players. We will use the deletion of a player in an augmenting system; then this player is a loop player in the augmenting system considered. It will be applied in the induction proof of Theorem 4.7.

Definition 4.5. An allocation rule $\gamma: A S \rightarrow \bigcup_{n \geq 1} \mathbb{R}^{n}$ is loop-null if, for all $(N, v, \mathcal{F}) \in A S$ and for any loop player $i$ in $(N, \mathcal{F})$, we have $\gamma_{i}(N, v, \mathcal{F})=0$.

Proposition 4.6. The value $\alpha: A S \rightarrow \bigcup_{n \geq 1} \mathbb{R}^{n}$ is loop-null on $A S$.

Proof. Given $(N, v, \mathcal{F}) \in A S$ and a loop player $i \in N$, we have $C_{\mathcal{F}}(S)=C_{\mathcal{F}}(S \backslash i)$ for all $S \subseteq N$. Then $v^{\mathcal{F}}(S)-v^{\mathcal{F}}(S \backslash i)=0$ for all $S \subseteq N$, and hence $\alpha_{i}(N, v, \mathcal{F})=$ $\Phi_{i}\left(N, v^{\mathcal{F}}\right)=0$.

For the study of the balanced contributions axiom, we will analyze the effect of deletion of a player, inspired by the contributions of Myerson [11] and Slikker [14].

Given a set system $(N, \mathcal{F})$ and $i \in N$, we define $\mathcal{F} \backslash i=\{S \in \mathcal{F}: i \notin S\}$. The set system $(N \backslash i, \mathcal{F} \backslash i)$ is the deletion of $i$ in $(N, \mathcal{F})$.

Proposition 4.7. If $(N, \mathcal{F})$ is an augmenting system and $i \in N$, then $(N \backslash i, \mathcal{F} \backslash i)$ is an augmenting system.

Proof. The set system $(N \backslash i, \mathcal{F} \backslash i)$ satisfies that $\emptyset \in \mathcal{F} \backslash i$. If $S, T \in \mathcal{F} \backslash i$ with $S \cap T \neq \emptyset$, then $S \cup T \in \mathcal{F}$ and $i \notin S \cup T$; hence $S \cup T \in \mathcal{F} \backslash i$. Finally, if $S, T \in \mathcal{F} \backslash i$ with $S \subset T$, there exists $j \in T \backslash S$ such that $S \cup j \in \mathcal{F}$. Since $T \in \mathcal{F} \backslash i$ implies $j \neq i$ and $i \notin S$, we conclude that $S \cup j \in \mathcal{F} \backslash i$.

Notice that if $(N, \mathcal{F})$ is an augmenting system and $i \in N$, then $(N, \mathcal{F} \backslash i)$ is also an augmenting system in which $i$ is a loop player.

Let $\gamma$ be an allocation rule on $A S$ and let $(N, v, \mathcal{F}) \in A S$. For all $i, j \in N$ with $i \neq j$, the contribution of player $i$ to the payoff of player $j$ is given by

$$
D_{i j}^{\gamma}(N, v, \mathcal{F})=\gamma_{j}(N, v, \mathcal{F})-\gamma_{j}(N, v, \mathcal{F} \backslash i) .
$$

DeFINITION 4.8. An allocation rule $\gamma$ on $A S$ has balanced contributions if, for every $(N, v, \mathcal{F}) \in A S$ and any two players $i, j \in N$ with $i \neq j$, we have

$$
D_{i j}^{\gamma}(N, v, \mathcal{F})=D_{j i}^{\gamma}(N, v, \mathcal{F}) \text {. }
$$

Copyright (c) by SIAM. Unauthorized reproduction of this article is prohibited. 
We prove that the value $\alpha$ on $A S$ has balanced contributions. First we introduce the following concepts. For every nonempty $R \subseteq N$, we define the unanimity game

$$
u_{R}(T)= \begin{cases}1 & \text { if } R \subseteq T \\ 0 & \text { otherwise }\end{cases}
$$

Every game is a unique linear combination of unanimity games (cf. Shapley [13]),

$$
v=\sum_{\{R \subseteq N: R \neq \emptyset\}} d_{R}(v) u_{R}, \text { where } d_{R}(v)=\sum_{T \subseteq R}(-1)^{|R|-|T|} v(T) .
$$

We shall call $d_{R}(v)$ the unanimity coefficient of $R$ in the game $v$. Owen [12] showed the following property: The unanimity games $u_{R}$, where $R$ is connected in the graph $G$, form a basis of the graph-restricted games. Let $(N, \mathcal{F})$ be the system of connected subgraphs of a graph $G=(N, E)$. Hamiache [7] proved a formula for computing the unanimity coefficients in the game $v^{\mathcal{F}}$ by using the outputs in the original game $v$. Applying the Möbius inversion formula, Bilbao [2] extended Hamiache's formula and Owen's property to the case in which $(N, \mathcal{F})$ is an augmenting system.

Proposition 4.9. Let $(N, v, \mathcal{F})$ be an augmenting structure. Then the restricted game $\left(N, v^{\mathcal{F}}\right)$ satisfies $v^{\mathcal{F}}=\sum_{\{R \in \mathcal{F}: R \neq \emptyset\}} d_{R}\left(v^{\mathcal{F}}\right) u_{R}$, where the unanimity coefficient

$$
d_{R}\left(v^{\mathcal{F}}\right)=\sum_{\left\{T \in \mathcal{F}: T \subseteq R \subseteq T^{+}\right\}}(-1)^{|R|-|T|} v(T)
$$

for every nonempty $R \in \mathcal{F}$ and $d_{R}\left(v^{\mathcal{F}}\right)=0$ for all $R \notin \mathcal{F}$.

LEMMA 4.10. If $(N, v, \mathcal{F})$ is an augmenting structure and $i \in N$, then the unanimity coefficients satisfy $d_{R}\left(v^{\mathcal{F}}\right)=d_{R}\left(v^{\mathcal{F} \backslash i}\right)$ for all $R \in \mathcal{F} \backslash i$.

Proof. If $R \in \mathcal{F} \backslash i$, then every $T \in \mathcal{F}$ such that $T \subseteq R$ satisfies $i \notin T$, and hence $T \in \mathcal{F} \backslash i$. As a consequence we obtain

$$
\left\{T \in \mathcal{F}: T \subseteq R \subseteq T^{+}\right\}=\left\{T \in \mathcal{F} \backslash i: T \subseteq R \subseteq T^{+}\right\},
$$

and Proposition 4.9 implies that $d_{R}\left(v^{\mathcal{F}}\right)=d_{R}\left(v^{\mathcal{F} \backslash i}\right)$.

The Shapley value is a linear mapping with respect to the characteristic function, and the images of the unanimity games are given by (cf. Owen [12])

$$
\Phi_{i}\left(N, u_{R}\right)= \begin{cases}1 /|R| & \text { if } i \in R \\ 0 & \text { otherwise }\end{cases}
$$

In terms of the unanimity coefficients $d_{R}\left(v^{\mathcal{F}}\right)$ in game $v^{\mathcal{F}}$, we have that

$$
\Phi_{i}\left(N, v^{\mathcal{F}}\right)=\sum_{\{R \in \mathcal{F}: i \in R\}} \frac{d_{R}\left(v^{\mathcal{F}}\right)}{|R|} .
$$

Next we compute the contribution of player $i \in N$ to the payoff of player $j \neq i$.

Proposition 4.11. For all $(N, v, \mathcal{F}) \in A S$ and all $i, j \in N$ with $i \neq j$, the allocation rule $\alpha$ satisfies

$$
D_{i j}^{\alpha}(N, v, \mathcal{F})=\sum_{\{R \in \mathcal{F}: i, j \in R\}} \frac{d_{R}\left(v^{\mathcal{F}}\right)}{|R|},
$$

and hence $\alpha$ has balanced contributions. 
Proof. Let us consider $i \in N, j \in N \backslash i$, and $\mathcal{F}_{i}=\{S \in \mathcal{F}: i \in S\}$. Since $\alpha(N, v, \mathcal{F})=\Phi\left(N, v^{\mathcal{F}}\right)$, applying Lemma 4.10 we obtain

$$
\begin{aligned}
D_{i j}^{\alpha}(N, v, \mathcal{F}) & =\Phi_{j}\left(N, v^{\mathcal{F}}\right)-\Phi_{j}\left(N, v^{\mathcal{F} \backslash i}\right) \\
& =\sum_{\{R \in \mathcal{F}: j \in R\}} \frac{d_{R}\left(v^{\mathcal{F}}\right)}{|R|}-\sum_{\{R \in \mathcal{F} \backslash i: j \in R\}} \frac{d_{R}\left(v^{\mathcal{F} \backslash i}\right)}{|R|} \\
& =\sum_{\left\{R \in \mathcal{F}_{i}: j \in R\right\}} \frac{d_{R}\left(v^{\mathcal{F}}\right)}{|R|} \\
& =\sum_{\{R \in \mathcal{F}: i, j \in R\}} \frac{d_{R}\left(v^{\mathcal{F}}\right)}{|R|},
\end{aligned}
$$

since $\left\{R \in \mathcal{F}_{i}: j \in R\right\}=\{R \in \mathcal{F}: i, j \in R\}$ implies the last equation.

The following theorem provides a characterization of the value $\alpha$ for augmenting structures.

THEOREM 4.12. The value $\alpha$ is the unique allocation rule on the set of all augmenting structures $A S$ that satisfies component efficiency, loop-null, and balanced contributions.

Proof. Propositions 4.4, 4.6, and 4.11 state that the value $\alpha$ satisfies component effiency, loop-null, and balanced contribution properties. Let us consider an allocation rule $\gamma$ on $A S$ which satisfies these three properties. We will prove that $\gamma(N, v, \mathcal{F})=$ $\alpha(N, v, \mathcal{F})$ for all $(N, v, \mathcal{F}) \in A S$ by induction on $k=|\mathcal{F}| \geq 1$.

If $k=1$, then $\mathcal{F}=\{\emptyset\}$, and hence any player $i \in N$ is a loop player. In view of the loop-null property, $\gamma_{i}(N, v, \mathcal{F})=\alpha_{i}(N, v, \mathcal{F})=0$ for all $i \in N$.

Let $k>1$ and suppose that $\gamma(N, v, \mathcal{F})=\alpha(N, v, \mathcal{F})$ for all $(N, v, \mathcal{F}) \in A S$ such that $|\mathcal{F}| \leq k-1$. We now consider an augmenting structure $(N, v, \mathcal{F})$ with $|\mathcal{F}|=k$. Since $N$ is the disjoint union of the set of loop players and the components of $N$, it suffices to prove that for every $M \in C_{\mathcal{F}}(N)$ we have $\gamma_{i}(N, v, \mathcal{F})=\alpha_{i}(N, v, \mathcal{F})$ for all $i \in M$.

If we suppose that $|M|=1$, applying component efficiency we obtain the equality. Then, without loss of generality, we denote $M=\{1, \ldots, m\} \in C_{\mathcal{F}}(N)$, where $m \geq 2$. Since $\gamma$ satisfies balanced contributions, we apply this property to pairs $\{1, j\}$, where $j=2, \ldots, m$, and hence we obtain the following system of $m$ equations:

$$
\begin{aligned}
\gamma_{2}(N, v, \mathcal{F})-\gamma_{1}(N, v, \mathcal{F}) & =\gamma_{2}(N, v, \mathcal{F} \backslash 1)-\gamma_{1}(N, v, \mathcal{F} \backslash 2), \\
& \vdots \\
\gamma_{m}(N, v, \mathcal{F})-\gamma_{1}(N, v, \mathcal{F}) & =\gamma_{m}(N, v, \mathcal{F} \backslash 1)-\gamma_{1}(N, v, \mathcal{F} \backslash m), \\
\sum_{i=1}^{m} \gamma_{i}(N, v, \mathcal{F}) & =v(M),
\end{aligned}
$$

where the last equation follows from the component efficiency property. We can use the induction hypothesis to obtain the following equivalent system of $m$ linear equations with $\gamma_{i}(N, v, \mathcal{F}), i \in M$, as unknowns:

$$
\begin{aligned}
\gamma_{2}(N, v, \mathcal{F})-\gamma_{1}(N, v, \mathcal{F}) & =\alpha_{2}(N, v, \mathcal{F} \backslash 1)-\alpha_{1}(N, v, \mathcal{F} \backslash 2), \\
& \vdots \\
\gamma_{m}(N, v, \mathcal{F})-\gamma_{1}(N, v, \mathcal{F}) & =\alpha_{m}(N, v, \mathcal{F} \backslash 1)-\alpha_{1}(N, v, \mathcal{F} \backslash m), \\
\sum_{i=1}^{m} \gamma_{i}(N, v, \mathcal{F}) & =v(M) .
\end{aligned}
$$

Copyright $@$ by SIAM. Unauthorized reproduction of this article is prohibited. 
The columns of the matrix of this system are linearly independent, and hence the system has a unique solution. Since the value $\alpha$ satisfies the balanced contribution and component efficiency properties, the vector $\left(\alpha_{1}(N, v, \mathcal{F}), \ldots, \alpha_{m}(N, v, \mathcal{F})\right)$ is a solution, and thus $\gamma_{i}(N, v, \mathcal{F})=\alpha_{i}(N, v, \mathcal{F})$ for all $i \in M$.

5. Consistency. An allocation rule $\gamma$ on the set of all games satisfies Hart and Mas-Colell consistency [8] if, when $\gamma$ is applied to a reduced game defined according to $\gamma$, it yields the same allocations as the original game. We will analyze this type of consistency on the set of all augmenting structures $A S$. Note that $\alpha$ is the Shapley value of a restricted game. A natural approach would be to reduce both the game and the restriction system. This approach will be carried out in the remainder of this section.

Given an augmenting system $(N, \mathcal{F})$ and a coalition $R \subseteq N$, we define the restriction system $\left(R, \mathcal{F}_{R}\right)$, where $\mathcal{F}_{R}=\{S \in \mathcal{F}: S \subseteq R\}$. It is clear that $\left(R, \mathcal{F}_{R}\right)$ is an augmenting system for all $R \subseteq N$. Note also that $\mathcal{F}_{\emptyset}=\{\emptyset\}, \mathcal{F}_{N}=\mathcal{F}$, and $\mathcal{F}_{N \backslash i}=\mathcal{F} \backslash i$. Furthermore, we define the trace on $R$ by $\mathcal{F}^{R}=\{S \cap R: S \in \mathcal{F}\}$. It is easy to verify that $\left(R, \mathcal{F}^{R}\right)$ is an augmenting system for all $R \subseteq N$. Note also that $\mathcal{F}^{N \backslash T}=\{S \backslash T: S \in \mathcal{F}\}$. We will write for simplicity $\mathcal{F}^{-T}$ and $\mathcal{F}^{-i}$ instead of $\mathcal{F}^{N \backslash T}$ and $\mathcal{F}^{N \backslash i}$. So, the restriction system $\mathcal{F}_{R}$ describes the cooperation possibilities that players in $R$ have without the help of players outside $R$. On the other hand, the trace $\mathcal{F}^{R}=\mathcal{F}^{-(N \backslash R)}$ describes the cooperation possibilities of players in $R$ under the assumption that the players outside $R$ cooperate.

Let us consider $\emptyset \neq T \subseteq N$ and $S \subseteq N \backslash T$. Then we define the set of augmenting players throughout $S$ as

$\overline{T_{S}}=\{i \in T$ : there exist $\emptyset \neq R \subseteq S$ and $Q \subseteq T$ with $i \in Q$ and $R \cup Q \in \mathcal{F}\}$.

Definition 5.1. Let $\gamma$ be an allocation rule on $A S$, let $(N, v, \mathcal{F}) \in A S$, and let $\emptyset \neq T \subseteq N$. The $\gamma$-reduced game $\left(N \backslash T, v_{\gamma}^{-T}\right)$ is given by

$$
v_{\gamma}^{-T}(S)=v\left(S \cup \overline{T_{S}}\right)-\sum_{i \in \overline{T_{S}}} \gamma_{i}\left(S \cup \overline{T_{S}}, v, \mathcal{F}_{S \cup \overline{T_{S}}}\right)
$$

for all $S \subseteq N \backslash T$.

Consistency is then defined as follows.

Definition 5.2. An allocation rule $\gamma$ on $A S$ is consistent if, for every $(N, v, \mathcal{F}) \in$ $A S$ and every $\emptyset \neq T \subseteq N$, we have that

$$
\gamma_{j}\left(N \backslash T, v_{\gamma}^{-T}, \mathcal{F}^{-T}\right)=\gamma_{j}(N, v, \mathcal{F}) \text { for all } j \in N \backslash T .
$$

We also define 1-consistency as consistency for any $T$ such that $|T|=1$. In this case, $\overline{T_{S}}=\{i \in T$ : there exists $\emptyset \neq R \subseteq S$ such that $R \cup i \in \mathcal{F}\}$. In order to analyze 1-consistency of the allocation rule $\alpha$, we prove the following lemmas.

Lemma 5.3. Let $(N, v, \mathcal{F}) \in A S$ and $i \in N$. Then for every $S \subseteq N \backslash i$

$$
r_{S}=c_{S}+\frac{|S|}{|S|+1} c_{S \cup i}
$$

holds, where $c_{S}$ and $r_{S}$ denote unanimity coefficients of $S$ in games $v^{\mathcal{F}}$ and $\left(v_{\alpha}^{-i}\right)^{\mathcal{F}^{-i}}$, respectively. 
Proof. Let $S \subseteq N \backslash i$. Since $\overline{\{i\}_{S}}=\{i\}$ if there exists $R \subseteq S$ such that $R \cup i \in \mathcal{F}$, and $\overline{\{i\}_{S}}=\emptyset$ otherwise, we have that

$$
v_{\alpha}^{-i}(S)= \begin{cases}v(S \cup i)-\alpha_{i}\left(S \cup i, v, \mathcal{F}_{S \cup i}\right) & \text { if } \exists R \subseteq S \text { with } R \cup i \in \mathcal{F}, \\ v(S) & \text { otherwise. }\end{cases}
$$

Now we compute the worth of the restricted game

$$
\left(v_{\alpha}^{-i}\right)^{\mathcal{F}^{-i}}(S)=\sum_{T \in C_{\mathcal{F}-i}(S)} v_{\alpha}^{-i}(T)
$$

We analyze the following two cases:

(a) If there exists $R \subseteq S$ with $R \cup i \in \mathcal{F}$, then $R \in \mathcal{F}^{-i}$ and we select the component $T^{*} \in C_{\mathcal{F}^{-i}}(S)$ such that $R \subseteq T^{*}$. Moreover, if $T \in C_{\mathcal{F}^{-i}}(S)$ with $T \neq T^{*}$, then $R^{\prime} \cup i \notin \mathcal{F}$ for all $R^{\prime} \subseteq T$, since otherwise $(R \cup i) \cap\left(R^{\prime} \cup i\right) \neq \emptyset$ implies $R \cup R^{\prime} \in$ $\mathcal{F}^{-i}$, contradicting the maximal feasibility of $T^{*}$ (or $T$ ). It follows from the definition that

$$
\begin{aligned}
& \left(v_{\alpha}^{-i}\right)^{\mathcal{F}^{-i}}(S)=v_{\alpha}^{-i}\left(T^{*}\right)+\sum_{\left\{T \in C_{\mathcal{F}}^{-i}(S): T \neq T^{*}\right\}} v_{\alpha}^{-i}(T) \\
& =v\left(T^{*} \cup i\right)-\alpha_{i}\left(T^{*} \cup i, v, \mathcal{F}_{T^{*} \cup i}\right)+\sum_{\left\{T \in C_{\mathcal{F}-i}(S): T \neq T^{*}\right\}} v(T) \\
& =\sum_{T \in C_{\mathcal{F}}(S \cup i)} v(T)-\alpha_{i}\left(T^{*} \cup i, v, \mathcal{F}_{T^{*} \cup i}\right) \\
& =v^{\mathcal{F}}(S \cup i)-\Phi_{i}\left(T^{*} \cup i, v^{\mathcal{F}_{T^{*} \cup i}}\right),
\end{aligned}
$$

where $\Phi_{i}$ denotes the Shapley value of player $i$. It follows from formula (1) that

$$
\Phi_{i}\left(T^{*} \cup i, v^{\mathcal{F}_{T^{*} \cup i}}\right)=\sum_{\left\{Q \in \mathcal{F}_{T^{*} \cup i}: i \in Q\right\}} \frac{d_{Q}\left(v^{\mathcal{F}_{T^{*} \cup i}}\right)}{|Q|} .
$$

By definition $\mathcal{F}_{T^{*} \cup i}=\left\{Q \in \mathcal{F}: Q \subseteq T^{*} \cup i\right\}$ and hence $v^{\mathcal{F}_{T^{*} \cup i}}=v^{\mathcal{F}}$ in $2^{\mathcal{F}_{T^{*} \cup i}}$. Moreover, $\left\{Q \in \mathcal{F}_{T^{*} \cup i}: i \in Q\right\}=\left\{P \cup i \in \mathcal{F}: P \subseteq T^{*}\right\}$. From this and Proposition 4.9 it then follows that

$$
\begin{aligned}
\Phi_{i}\left(T^{*} \cup i, v^{\mathcal{F}_{T^{*} \cup i}}\right) & =\sum_{\left\{P \subseteq T^{*}: P \cup i \in \mathcal{F}\right\}} \frac{d_{P \cup i}\left(v^{\mathcal{F}}\right)}{|P|+1} \\
& =\sum_{P \subseteq T^{*}} \frac{d_{P \cup i}\left(v^{\mathcal{F}}\right)}{|P|+1} \\
& =\sum_{P \subseteq S} \frac{d_{P \cup i}\left(v^{\mathcal{F}}\right)}{|P|+1}
\end{aligned}
$$

and therefore

$$
\left(v_{\alpha}^{-i}\right)^{\mathcal{F}^{-i}}(S)=v^{\mathcal{F}}(S \cup i)-\sum_{P \subseteq S} \frac{c_{P \cup i}}{|P|+1} .
$$

Copyright $@$ by SIAM. Unauthorized reproduction of this article is prohibited. 
We will finish our proof of the case (a) by an induction argument. Let us consider $S \subseteq N \backslash i$ with $|S|=1$. Then

$$
\begin{aligned}
r_{S} & =\left(v_{\alpha}^{-i}\right)^{\mathcal{F}^{-i}}(S)=v^{\mathcal{F}}(S \cup i)-\sum_{P \subseteq S} \frac{c_{P \cup i}}{|P|+1} \\
& =c_{S}+c_{\{i\}}+c_{S \cup i}-\left(c_{\{i\}}+\frac{1}{2} c_{S \cup i}\right) \\
& =c_{S}+\frac{|S|}{|S|+1} c_{S \cup i} .
\end{aligned}
$$

Let $k \geq 1$ and suppose the statement is true for all coalitions of size at most $k$. Let $S \subseteq N \backslash i$ with $|S|=k+1$. Since

$$
\left(v_{\alpha}^{-i}\right)^{\mathcal{F}^{-i}}(S)=\sum_{P \subseteq S} r_{P}=r_{S}+\sum_{P \subset S} r_{P}
$$

we obtain

$$
\begin{aligned}
r_{S} & =v^{\mathcal{F}}(S \cup i)-\sum_{P \subseteq S} \frac{c_{P \cup i}}{|P|+1}-\sum_{P \subseteq S} r_{P} \\
& =\sum_{P \subseteq S \cup i} c_{P}-\sum_{P \subseteq S} \frac{c_{P \cup i}}{|P|+1}-\sum_{P \subseteq S}\left(c_{P}+\frac{|P|}{|P|+1} c_{P \cup i}\right) \\
& =\sum_{P \subseteq S}\left(c_{P}+c_{P \cup i}\right)-\sum_{P \subseteq S} \frac{c_{P \cup i}}{|P|+1}-\sum_{P \subseteq S}\left(c_{P}+\frac{|P|}{|P|+1} c_{P \cup i}\right) \\
& =c_{S}+\frac{|S|}{|S|+1} c_{S \cup i} .
\end{aligned}
$$

(b) If $R \cup i \notin \mathcal{F}$, then for all $R \subseteq S$ every $T \in C_{\mathcal{F}^{-i}}(R)$ satisfies $T \in \mathcal{F}^{-i}$ and $T \cup i \notin \mathcal{F}$, which implies that $T \in \mathcal{F}$. As a consequence

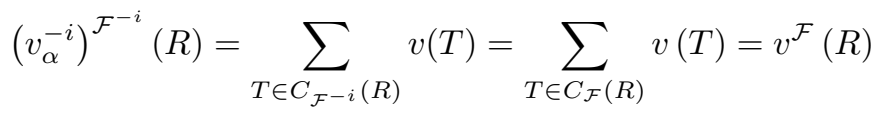

for every $R \subseteq S$, and hence

$$
r_{S}=c_{S}=c_{S}+\frac{|S|}{|S|+1} c_{S \cup i}
$$

where the last equation follows from $c_{S \cup i}=0$.

The following result states that $\alpha$ satisfies 1-consistency.

Lemma 5.4. The allocation rule $\alpha$ satisfies 1-consistency.

Proof. Let $(N, v, \mathcal{F}) \in A S$ and let $i \in N$. We denote by $c_{S}$ and $r_{S}$ the unanimity coefficients of $S \subseteq N \backslash i$ in games $v^{\mathcal{F}}$ and $\left(v_{\alpha}^{-i}\right)^{\mathcal{F}^{-i}}$, respectively. By using the 
representation of the Shapley value in terms of unanimity coefficients and Lemma 5.3 , we obtain

$$
\begin{aligned}
\alpha_{j}\left(N \backslash i, v_{\alpha}^{-i}, \mathcal{F}^{-i}\right) & =\Phi_{j}\left(N \backslash i,\left(v_{\alpha}^{-i}\right)^{\mathcal{F}^{-i}}\right) \\
& =\sum_{\{S \subseteq N \backslash i: j \in S\}} \frac{r_{S}}{|S|} \\
& =\sum_{\{S \subseteq N \backslash i: j \in S\}} \frac{c_{S}+\frac{|S|}{|S|+1} c_{S \cup i}}{|S|} \\
& =\sum_{\{T \subseteq N: j \in T\}} \frac{c_{T}}{|T|} \\
& =\alpha_{j}(N, v, \mathcal{F})
\end{aligned}
$$

for all $j \in N \backslash i$.

THEOREM 5.5. The allocation rule $\alpha$ satisfies consistency.

Proof. We will show that for all augmenting structures $(N, v, \mathcal{F})$ and all nonempty $T \subset N$, the equality $\alpha_{j}(N, v, \mathcal{F})=\alpha_{j}\left(N \backslash T, v_{\alpha}^{-T}, \mathcal{F}^{-T}\right)$ holds for all $j \in N \backslash T$. We proceed by induction on $|T|$. By Lemma 5.4 the statement is true for $|T|=1$. Let $k \geq 1$ and suppose the statement is true for all $T \subset N$ with $|T| \leq k$. Now consider $T \subset N$ with $|T|=k$; we show that it is also true for $T \cup i$, where $i \in N \backslash T$. Suppose $S \subseteq N \backslash(T \cup i)$ and consider the following two cases:

(a) If there exists $R \subseteq S$ with $R \cup i \in \mathcal{F}^{-T}$, then

$$
\begin{aligned}
\left(v_{\alpha}^{-T}\right)_{\alpha}^{-i}(S)= & v_{\alpha}^{-T}(S \cup i)-\alpha_{i}\left(S \cup i, v_{\alpha}^{-T},\left(\mathcal{F}^{-T}\right)_{S \cup i}\right) \\
= & v\left(S \cup i \cup \overline{T_{S \cup i}}\right)-\sum_{j \in \overline{T_{S \cup i}}} \alpha_{j}\left(S \cup i \cup \overline{T_{S \cup i}}, v, \mathcal{F}_{S \cup i \cup \overline{T_{S \cup i}}}\right) \\
& -\alpha_{i}\left(S \cup i, v_{\alpha}^{-T},\left(\mathcal{F}^{-T}\right)_{S \cup i}\right) \\
= & v\left(S \cup i \cup \overline{T_{S \cup i}}\right)-\sum_{j \in \overline{T_{S \cup i}}} \alpha_{j}\left(S \cup i \cup \overline{T_{S \cup i}}, v, \mathcal{F}_{S \cup i \cup \overline{T_{S \cup i}}}\right) \\
& -\alpha_{i}\left(S \cup i \cup T, v, \mathcal{F}_{S \cup i \cup T}\right) \\
= & v\left(S \cup i \cup \overline{T_{S \cup i}}\right)-\sum_{j \in \overline{T_{S \cup i}}} \alpha_{j}\left(S \cup i \cup \overline{T_{S \cup i}}, v, \mathcal{F}_{S \cup i \cup \overline{T_{S \cup i}}}\right) \\
& -\alpha_{i}\left(S \cup i \cup \overline{T_{S \cup i}}, v, \mathcal{F}_{S \cup i \cup \overline{T_{S \cup i}}}\right) \\
= & v\left(S \cup \overline{(T \cup i)_{S}}\right)-\sum_{j \in\left(\overline{T \cup i)_{S}}\right.} \alpha_{j}\left(S \cup \overline{(T \cup i)_{S}}, v, \mathcal{F}_{S \cup \overline{(T \cup i)_{S}}}\right) \\
= & v_{\alpha}^{-(T \cup i)}(S) .
\end{aligned}
$$

The first two equalities follow by definition. The third follows by the induction hypothesis and since $\left(\mathcal{F}^{-T}\right)_{S \cup i}=\left(\mathcal{F}_{S \cup i \cup T}\right)^{-T}$. The fourth equality holds since players in $T \backslash \overline{T_{S \cup i}}$ do not influence the payoff of player $i$. By condition (a) there exists $R \subseteq S$ and $Q \subseteq T$ such that $R \cup Q \cup i \in \mathcal{F}$, which implies $i \in \overline{(T \cup i)_{S}}$. Indeed, $\overline{T_{S \cup i}} \cup i=\overline{(T \cup i)_{S}}$, and hence the fifth equality holds. The last equality holds by definition. 
(b) If $R \cup i \notin \mathcal{F}^{-T}$ for all $R \subseteq S$, then

$$
\begin{aligned}
\left(v_{\alpha}^{-T}\right)_{\alpha}^{-i}(S) & =v_{\alpha}^{-T}(S) \\
& =v\left(S \cup \overline{T_{S}}\right)-\sum_{j \in \overline{T_{S}}} \alpha_{j}\left(S \cup \overline{T_{S}}, v, \mathcal{F}_{S \cup \overline{T_{S}}}\right) \\
& =v\left(S \cup \overline{(T \cup i)_{S}}\right)-\sum_{j \in \overline{(T \cup i)_{S}}} \alpha_{j}\left(S \cup \overline{(T \cup i)_{S}}, v, \mathcal{F}_{S \cup \overline{(T \cup i)_{S}}}\right) \\
& =v_{\alpha}^{-(T \cup i)}(S) .
\end{aligned}
$$

The first two equalities follow by definition. The third equality holds since $\overline{T_{S}}=$ $\overline{(T \cup i)_{S}}$, because of condition (b), implies that $R \cup Q \cup i \notin \mathcal{F}$ for all $R \subseteq S$ and all $Q \subseteq T$. The last equality holds by definition.

Using the result of these two cases, we get

$$
\begin{aligned}
\alpha_{j}(N, v, \mathcal{F}) & =\alpha_{j}\left(N \backslash T, v_{\alpha}^{-T}, \mathcal{F}^{-T}\right) \\
& =\alpha_{j}\left((N \backslash T) \backslash i,\left(v_{\alpha}^{-T}\right)_{\alpha}^{-i},\left(\mathcal{F}^{-T}\right)^{-i}\right) \\
& =\alpha_{j}\left(N \backslash(T \cup i),\left(v_{\alpha}^{-T}\right)_{\alpha}^{-i}, \mathcal{F}^{-(T \cup i)}\right) \\
& =\alpha_{j}\left(N \backslash(T \cup i), v_{\alpha}^{-(T \cup i)}, \mathcal{F}^{-(T \cup i)}\right)
\end{aligned}
$$

for all $j \in N \backslash(T \cup i)$, where the first two equalities hold by the induction hypothesis and 1-consistency, respectively. The third equality holds since

$$
\mathcal{F}^{-(T \cup i)}=\{S \backslash(T \cup i): S \in \mathcal{F}\}=\left\{(S \backslash T) \backslash i: S \backslash T \in \mathcal{F}^{-T}\right\}=\left(\mathcal{F}^{-T}\right)^{-i} .
$$

The last equality follows by the result derived from the two cases. We conclude that the statement in the theorem is true for all $(N, v, \mathcal{F})$ and all $T \subset N$ with $|T|=$ $k+1$.

We will obtain a characterization of the value $\alpha$ by using consistency and the initial condition for two-person games defined as follows.

DeFINITION 5.6. An allocation rule $\gamma$ on $A S$ is standard for two-person restricted games if for all $(N, v, \mathcal{F}) \in A S$ with $N=\{i, j\}$ and all $k \in N$ it holds that

$$
\gamma_{k}(N, v, \mathcal{F})=v^{\mathcal{F}}(\{k\})+\frac{1}{2}\left[v^{\mathcal{F}}(N)-v^{\mathcal{F}}(\{i\})-v^{\mathcal{F}}(\{j\})\right] .
$$

THEOREM 5.7. The value $\alpha$ is the unique allocation rule on AS that satisfies consistency and is the standard property for two-person restricted games.

Proof. By Theorem 5.5 we know that $\alpha$ satisfies consistency. We will first show that the value $\alpha$ is standard as well. Since the Shapley value is standard, we find that

$$
\alpha_{k}(N, v, \mathcal{F})=\Phi_{k}\left(N, v^{\mathcal{F}}\right)=v^{\mathcal{F}}(\{k\})+\frac{1}{2}\left[v^{\mathcal{F}}(N)-v^{\mathcal{F}}(\{i\})-v^{\mathcal{F}}(\{j\})\right]
$$

for all $k \in N$, and all $(N, v, \mathcal{F}) \in A S^{N}$ such that $N=\{i, j\}$.

For the converse, assume that the allocation rule $\gamma$ on $A S$ is consistent and standard. We show first, by using induction on $|N|$, that $\gamma$ satisfies component efficiency. The standard property yields clearly that $\gamma$ satisfies the component efficiency property for all $(N, v, \mathcal{F}) \in A S^{N}$ such that $|N|=2$. 
For $|N|=1$, we have to show that

$$
\gamma_{i}(\{i\}, v, \mathcal{F})=v^{\mathcal{F}}(\{i\})= \begin{cases}0 & \text { if } \mathcal{F}=\{\emptyset\}, \\ v(\{i\}) & \text { if } \mathcal{F}=\{\emptyset,\{i\}\} .\end{cases}
$$

Consider $(\{i, j\}, w, \mathcal{F}) \in A S$, where $w(\{i\})=w(\{i, j\})=v(\{i\})$, and $w(\{j\})=$ 0 . Since $\{j\} \notin \mathcal{F}$ and $\{i, j\} \notin \mathcal{F}$, the standard property implies that $\gamma_{j}(\{i, j\}, w, \mathcal{F})=$ 0 and

$$
\begin{aligned}
\gamma_{i}(\{i, j\}, w, \mathcal{F}) & =w^{\mathcal{F}}(\{i\})+\frac{1}{2}\left[w^{\mathcal{F}}(\{i, j\})-w^{\mathcal{F}}(\{i\})-w^{\mathcal{F}}(\{j\})\right] \\
& =v^{\mathcal{F}}(\{i\})+\frac{1}{2}\left[v^{\mathcal{F}}(\{i\})-v^{\mathcal{F}}(\{i\})\right] \\
& =v^{\mathcal{F}}(\{i\}) .
\end{aligned}
$$

As $v=w_{\gamma}^{-j}$ and $\mathcal{F}^{-j}=\mathcal{F}$ we conclude by consistency of $\gamma$ that

$$
\gamma_{i}(\{i\}, v, \mathcal{F})=\gamma_{i}(\{i, j\}, w, \mathcal{F})=v^{\mathcal{F}}(\{i\}) .
$$

Let $k \geq 3$ and suppose that $\gamma$ is component efficient for all $(N, v, \mathcal{F}) \in A S$ with $|N| \leq k-1$. Let us consider $(N, v, \mathcal{F}) \in A S$ with $|N|=k$. For every component $S \in C_{\mathcal{F}}(N)$ we take $T=\{j\} \subseteq S$. Since $\gamma$ satisfies 1-consistency, we have

$$
\begin{aligned}
\sum_{i \in S} \gamma_{i}(N, v, \mathcal{F}) & =\sum_{i \in S \backslash T} \gamma_{i}\left(N \backslash T, v_{\gamma}^{-T}, \mathcal{F}^{-T}\right)+\gamma_{j}(N, v, \mathcal{F}) \\
& =v_{\gamma}^{-T}(S \backslash T)+\gamma_{j}(N, v, \mathcal{F}) \\
& =v(S)-\gamma_{j}\left(S, v, \mathcal{F}_{S}\right)+\gamma_{j}(N, v, \mathcal{F}) \\
& =v(S) .
\end{aligned}
$$

The first equality follows by 1-consistency. As $S \backslash T \in C_{\mathcal{F}^{-T}}(N \backslash T)$ the induction hypothesis implies the second equality. Since $\overline{T_{S \backslash T}}=T=\{j\}$, the third equality follows by definition and the last by consistency because of

$$
\gamma_{j}(N, v, \mathcal{F})=\gamma_{j}\left(S, v_{\gamma}^{-(N \backslash S)}, \mathcal{F}^{S}\right)=\gamma_{j}\left(S, v, \mathcal{F}_{S}\right)
$$

where $v_{\gamma}^{-(N \backslash S)}=v$ and $\mathcal{F}^{S}=\mathcal{F}_{S}$ are a consequence of the maximal feasibility of $S \in C_{\mathcal{F}}(N)$. Therefore, we conclude that $\gamma$ is component efficient.

We will show by induction on $n=|N|$ that $\gamma(N, v, \mathcal{F})=\alpha(N, v, \mathcal{F})$ for all $(N, v, \mathcal{F}) \in A S$. This relation has already been shown for $n=1$, whereas for $n=2$ the equality follows from the standard property for two-person restricted games. Let $n \geq 3$ and assume that the two allocation rules coincide for all augmenting structures with fewer than $n$ players. For every loop player $j \in N \backslash \bigcup_{S \in C_{\mathcal{F}}(N)} S$ we have by consistency

$$
\begin{aligned}
\gamma_{j}(N, v, \mathcal{F}) & =\gamma_{j}\left(\{j\}, v_{\gamma}^{-(N \backslash j)}, \mathcal{F}^{\{j\}}\right) \\
& =\gamma_{j}\left(\{j\}, v_{\gamma}^{-(N \backslash j)},\{\emptyset\}\right) \\
& =0 \\
& =\alpha_{j}(N, v, \mathcal{F}),
\end{aligned}
$$

Copyright $@$ by SIAM. Unauthorized reproduction of this article is prohibited. 
where the third equality follows by the induction hypothesis and the fourth equality holds by Proposition 4.6.

It remains to consider players that belong to a component. Consider $U \in C_{\mathcal{F}}(N)$. If $|U|=1$, the result holds by component efficiency, so we can only study the cases in which $|U|>1$. Let $T=\{i, j\} \subseteq U$ with $i \neq j$. Then for every $S \subseteq T$ such that $|S|=1$, the induction hypothesis implies

$$
\begin{aligned}
v_{\gamma}^{-(N \backslash T)}(S) & =v\left(S \cup \overline{(N \backslash T)_{S}}\right)-\sum_{k \in \overline{(N \backslash T)_{S}}} \gamma_{k}\left(S \cup \overline{(N \backslash T)_{S}}, v, \mathcal{F}_{S \cup \overline{(N \backslash T)_{S}}}\right) \\
& =v\left(S \cup \overline{(N \backslash T)_{S}}\right)-\sum_{k \in \overline{(N \backslash T)_{S}}} \alpha_{k}\left(S \cup \overline{(N \backslash T)_{S}}, v, \mathcal{F}_{S \cup \overline{(N \backslash T)_{S}}}\right) \\
& =v_{\alpha}^{-(N \backslash T)}(S) .
\end{aligned}
$$

Denote $w_{\gamma}=\left(v_{\gamma}^{-(N \backslash T)}\right)^{\mathcal{F}^{T}}$ and $w_{\alpha}=\left(v_{\alpha}^{-(N \backslash T)}\right)^{\mathcal{F}^{T}}$. Since $\gamma$ and $\alpha$ satisfy consistency and the standard property for two-person restricted games, we have

$$
\begin{aligned}
\gamma_{k}(N, v, \mathcal{F}) & =\gamma_{k}\left(T, v_{\gamma}^{-(N \backslash T)}, \mathcal{F}^{T}\right) \\
& =w_{\gamma}(\{k\})+\frac{1}{2}\left[w_{\gamma}(T)-w_{\gamma}(\{i\})-w_{\gamma}(\{j\})\right],
\end{aligned}
$$

and also

$$
\begin{aligned}
\alpha_{k}(N, v, \mathcal{F}) & =\alpha_{k}\left(T, v_{\alpha}^{-(N \backslash T)}, \mathcal{F}^{T}\right) \\
& =w_{\alpha}(\{k\})+\frac{1}{2}\left[w_{\alpha}(T)-w_{\alpha}(\{i\})-w_{\alpha}(\{j\})\right]
\end{aligned}
$$

for every $k \in\{i, j\}=T$. Therefore, using equality (2),

$$
\gamma_{i}(N, v, \mathcal{F})-\alpha_{i}(N, v, \mathcal{F})=\frac{1}{2}\left[w_{\gamma}(T)-w_{\alpha}(T)\right]=\gamma_{j}(N, v, \mathcal{F})-\alpha_{j}(N, v, \mathcal{F})
$$

for all $i, j \in T$. Thus, the above equality is true for any two players $i, j \in U$. Combining this with component efficiency of $\gamma$ and $\alpha$ yields

$$
\sum_{i \in U}\left[\gamma_{i}(N, v, \mathcal{F})-\alpha_{i}(N, v, \mathcal{F})\right]=v(U)-v(U)=0,
$$

which implies $\gamma_{i}(N, v, \mathcal{F})=\alpha_{i}(N, v, \mathcal{F})$ for all $i \in U$.

6. Computing directly the value $\alpha$. Finally, since we have studied two different approaches to characterize the value $\alpha$ from a theoretical point of view, we think that it might be interesting to give an algorithm written using Mathematica code to compute it directly and whose computational complexity is polynomial in the cardinality of the feasible coalitions. Notice that one of the main problems is to compute this value when the number of players is large. To provide this algorithm we based it on an explicit formula given by Bilbao [2].

Let $(N, v, \mathcal{F})$ be an augmenting structure. The value $\alpha(N, v, \mathcal{F})$ is given by

$$
\alpha_{i}(N, v, \mathcal{F})=\sum_{\{S \subseteq N: i \in S\}} \frac{(s-1) !(n-s) !}{n !}\left[v^{\mathcal{F}}(S)-v^{\mathcal{F}}(S \backslash i)\right],
$$

Copyright $@$ by SIAM. Unauthorized reproduction of this article is prohibited. 
where $i \in N, n=|N|$, and $s=|S|$. This value is an average of the marginal contributions $v^{\mathcal{F}}(S)-v^{\mathcal{F}}(S \backslash i)$ of a player $i$ to all coalitions $S \in 2^{N} \backslash\{\emptyset\}$.

If the number of players is $n$, then the function that measures the worst-case running time for computing this index is in $O\left(n 2^{n}\right)$. Moreover, to obtain the restricted game $v^{\mathcal{F}}$ we need to compute the set of the components $C_{\mathcal{F}}(S)$ of every subset $S \subseteq N$. Then it is necessary to consider all the feasible subsets of $S$, and hence the time complexity is $O(t)$, where

$$
t=\sum_{s=0}^{n}\left(\begin{array}{c}
n \\
s
\end{array}\right) 2^{s}=3^{n}
$$

As a consequence of Proposition 4.9, Bilbao [2] obtains the following explicit formula, in terms of $v$, for the Shapley value of the players in the restricted game $v^{\mathcal{F}}$. The time complexity of the formula is polynomial in the cardinality $|\mathcal{F}|$.

TheOREM 6.1. Let $(N, v, \mathcal{F})$ be an augmenting structure. Then

$$
\alpha_{i}(N, v, \mathcal{F})=\sum_{\{T \in \mathcal{F}: i \in T\}} \frac{(t-1) ! t^{*} !}{t^{+} !} v(T)-\sum_{\left\{T \in \mathcal{F}: i \in T^{*}\right\}} \frac{t !\left(t^{*}-1\right) !}{t^{+} !} v(T),
$$

where $i \in N, t=|T|, t^{*}=\left|T^{*}\right|$, and $t^{+}=\left|T^{+}\right|$.

The following algorithm obtained from Theorem 6.1, and written using Mathematica code (Wolfram [16]), computes the value $\alpha(N, v, \mathcal{F})$.

$<<$ DiscreteMath'Combinatorica'

Feasible [i_, F_List $]:=$ Feasible $[i, F]=\operatorname{Select}[F,($ MemberQ $[\#, i]) \&]$

SupInt [S_List,F_List] :=Select $[\mathrm{T},(\operatorname{MemberQ}[\mathrm{F}, \operatorname{Union}[\mathrm{S},\{\#\}]]) \&]$

Augmentation $\left[i_{-}, F_{\text {_List }}\right]:=$ Augmentation $[i, F]=\operatorname{Select}[\mathrm{F}$, (DeleteCases $[\#, i]==\#) \& \&($ MemberQ $[F, \operatorname{Union}[\#,\{i\}]]) \&]$

$\operatorname{co1}\left[\mathrm{S} \_L i s t\right]:=\operatorname{co} 1[\mathrm{~S}]=($ Length $[\mathrm{S}]-1) ! *$

(Length [SupInt [S,F] ]-Length [S] ) ! /Length [SupInt [S,F] ] ! ;

co2 [S_List] $:=\operatorname{co} 2[\mathrm{~S}]=\operatorname{co} 1[\mathrm{~S}] *$ Length $[\mathrm{S}] /$

(Length [SupInt $[\mathrm{S}, \mathrm{F}]]$-Length $[\mathrm{S}]$ );

AlphaValue [game_:Null] :=Module $[\{$ value $\}$, value=Table $[$ Apply $[P l u s$, If $[\#==\{\}, 0$, co1 [\#] (v[\#])]\& /@ Feasible $[\mathrm{i}, \mathrm{F}]]-$ Apply $[$ Plus, If $[\#==\{\}, 0, \operatorname{co} 2[\#] \mathrm{v}[\#]] \& / @$ Augmentation $[i, F]]$, $\{i$,Length [T] $\}]$; Return [value] ] ;

\section{REFERENCES}

[1] E. Algaba, J. M. Bilbao, R. van den Brink, and A. Jiménez-Losada, Cooperative games on antimatroids, Discrete Math., 282 (2004), pp. 1-15.

[2] J. M. BILBAO, Cooperative games under augmenting systems, SIAM J. Discrete Math., 17 (2003), pp. 122-133.

[3] P. Borm, G. Owen, And S. Tiss, On the position value for communication situations, SIAM J. Discrete Math., 5 (1992), pp. 305-320.

Copyright $@$ by SIAM. Unauthorized reproduction of this article is prohibited. 
[4] R. P. Dilworth, Lattices with unique irreducible decompositions, Ann. of Math. (2), 41 (1940), pp. $771-777$.

[5] P. H. Edelman and R. E. Jamison, The theory of convex geometries, Geom. Dedicata, 19 (1985), pp. 247-270.

[6] R. P. Gilles, G. Owen, and R. van den Brink, Games with permission structures: The conjunctive approach, Internat. J. Game Theory, 20 (1992), pp. 277-293.

[7] G. Hamiache, A value with incomplete communication, Games Econom. Behav., 26 (1999), pp. $59-78$.

[8] S. Hart and A. Mas-Colell, Potential, value and consistency, Econometrica, 57 (1989), pp. 589-614.

[9] B. Korte, L. Lovász, and R. Schrader, Greedoids, Springer, Berlin, 1991.

[10] R. B. Myerson, Graphs and cooperation in games, Math. Oper. Res., 2 (1977), pp. 225-229.

[11] R. B. Myerson, Conference structures and fair allocations rules, Internat. J. Game Theory, 9 (1980), pp. 169-182.

[12] G. Owen, Values of graph-restricted games, SIAM J. Algebraic Discrete Methods, 7 (1986), pp. 210-220.

[13] L. S. Shapley, A value for n-person games, in Contributions to the Theory of Games II, Ann. Math. Stud. 28, Princeton University Press, Princeton, NJ, 1953, pp. 307-317.

[14] M. Slikker, A characterization of the position value, Internat. J. Game Theory, 33 (2005), pp. $505-514$.

[15] R. VAN DEN BRINK, An axiomatization of the disjunctive permission value for games with a permission structure, Internat. J. Game Theory, 26 (1997), pp. 27-43.

[16] S. Wolfram, The Mathematica Book, 4th ed., Wolfram Media, Champaign, IL, Cambridge University Press, Cambridge, UK, 1999.

Copyright (C) by SIAM. Unauthorized reproduction of this article is prohibited. 